\title{
Portfolio rankings with skewness and kurtosis
}

\author{
M. Di Pierro ${ }^{1} \&$ J. Mosevich ${ }^{2}$ \\ ${ }^{1}$ DePaul University, School of Computer Science, \\ 243 S. Wabash Avenue, Chicago, IL 60604, USA \\ ${ }^{2}$ DePaul University, College of Commerce, 1 E. Jackson, \\ Chicago, IL 60604, USA
}

\begin{abstract}
In this paper we discuss the issue of portfolio ranking for a rational risk averse investor with and without the option to buy a risk free asset. We find that in the former case the use of Sharpe, Omega, Sortino, and Kappa rankings are all justified although they follow from different definitions. We also find that for portfolios with Gaussian distributed returns these rankings, as well as the Stutzer ranking, are equivalent to each other.

Finally we prove that without a risk free asset all the above rankings are incompatible with being a rational risk averse investor and a different ranking is required. We propose an exact analytical formula as well as an approximate formula for practical use.

Keywords: portfolio ranking, skewness, kurtosis, Omega, non-Gaussian disributions.
\end{abstract}

\section{Introduction}

In this paper we discuss the issue of portfolio ranking and selection. We will concentrate on selecting one portfolio among a finite set of portfolios, where each portfolio is characterized by its own distribution of returns $p(x)$. This distribution may be inferred from past performances and assumed to be persistent, or it may be derived by some model of future performances.

We distinguish two main cases:

CASE \#1 An amount $A$ must be invested and it can be distributed between a risk free asset (that pays a risk free rate $r$ ) and the selected portfolio.

CASE \#2 An amount $A$ must be invested exclusively in the selected portfolio. 
The two problems are apparently similar but they are conceptually different and therefore have different solutions. In this paper we wish to clarify the difference between the two, discuss ranking schemes in both cases, and propose a theoretically sound ranking scheme for the second case in the presence of distributions that exhibit arbitrary skewness and kurtosis.

We conclude that in case \#2, and in presence of skewness and kurtosis, a rational investor using the CARA utility function should select that portfolio with higher value of

$$
\mu-\frac{m \sigma^{2}}{2}+\frac{m^{2} \sigma^{3} S}{6}-\frac{m^{3} \sigma^{4}(K-3)}{720}
$$

where $\mu$ is the average return of the portfolio, $\sigma$ is the standard deviation, $S$ is the skewness, $K$ is the kurtosis, and $m$ is the CARA subjective risk aversion parameter.

Eq. (1) extends a previous conclusion by Levy and Markowitz [16] by taking into considerations the effects of skewness and kurtosis. Our formula provides a simple practical way to select one out of many mutually exclusive portfolios.

In this paper we discuss only the issue of portfolio selection, not portfolio construction. For a review, we refer the reader to the work of Ortobelli et al. [1].

In this analysis, we will always identify a portfolio with its distribution of returns $p$ since we are not interested in the composition of the portfolios.

\section{Portfolio selection}

In this section we discuss similarities and differences between CASE \#1 and \#2.

The main similarity between the two cases is that they both require subjective choice. In Utility Theory [3,4], this is the choice of a utility function $U(x)$ and its parameters. $U(x)$ is a function that takes as input a possible return $x$ from an investment and outputs a number that represents the investor's degree of satisfaction associated with a return $x$.

In neoclassical economics, an investor is defined "rational" if

- the investor has a utility function $U(x)$

- the investor acts in order to maximize $U(x)$

- $U(x)$ is monotonic increasing (higher return is preferred to lower return).

A common choice for the utility function is $U_{C A R A}(x) \equiv-e^{-m x}$ which is known as the "Constant Absolute Risk Averse" utility function. $m$ is the risk aversion parameter and it is of the order 1 .

We call a rational investor with a CARA utility function a "rational risk averse investor" or, more simply, "investor".

The investor will rank a portfolio $p$ by weighting the utility of a return $x$ with the probability of that return $p(x)$, thus he would use the ranking function

$$
R_{U}(p) \stackrel{\text { def }}{=} \int_{-\infty}^{+\infty} U(x) p(x) \mathrm{d} x
$$

or an equivalent function. 
Two rankings function, $R_{1}$ and $R_{2}$, are equivalent if and only if they produce the same ranking, i.e. if there is a monotonic function $h$ that maps $R_{2}(p)$ into $R_{1}(p)$ for every $p$. We will indicate the equivalence of two ranking functions with $R_{1} \sim R_{2}$.

\subsection{CASE \#1: with risk free asset}

In CASE \#1 our investor can choose to invest part of the funds $A$ into a risk free asset, for example a US Threasury-bill. This means that given any two portfolios $p_{1}$ and $p_{2}$ characterized by an average return and risk (standard deviation) $\mu_{1}, \sigma_{1}$ and $\mu_{2}, \sigma_{2}$ if

$$
\frac{\mu_{1}-r}{\sigma_{1}}>\frac{\mu_{2}-r}{\sigma_{2}}
$$

then the investor should never choose $p_{2}$ over $p_{1}$. This is because the investor can invest a fraction $\alpha=\sigma_{2} / \sigma_{1}$ of the total funds $A$ into portfolio $p_{1}$ and a fraction $(1-\alpha)$ into the risk free asset and obtain a new combined portfolio with the same risk as portfolio $p_{2}$ but a higher return, given by [6]

$$
\mu^{\prime}=(1-\alpha) r+\alpha \mu_{1}>\mu_{2}
$$

Hence portfolio $p_{1}$ is always preferable to portfolio $p_{2}$.

If one applies this argument to every portfolio in the set, one finds that our investor should invest part of the funds $A$ into the portfolio $p$ with the largest value of

$$
R_{\text {Sharpe }}(p) \stackrel{\text { def }}{=} \frac{\mu-r}{\sigma}
$$

This is the well known Sharpe ratio or Sharpe ranking function [7] [6].

The value of $\alpha$ is then determined by maximizing the utility function $U$. If the expected returns of the portfolios have a Gaussian distribution then

$$
\alpha=\alpha \text { that maximizes } \int U(\alpha x+(1-\alpha) r) p(x) \mathrm{d} x=\frac{\sqrt{\mu / r}}{m \sigma}
$$

If the return of the portfolios is not Gaussian distributed, the first equality in eq. (6) remains true, while the second equality is only an approximation since the integral must be performed numerically.

Similarly, if one adopts a definition of risk other than standard deviation of returns the argument that led to the $R_{\text {Sharpe }}$ measure remains valid, but $\sigma$ must be consistently replaced with the new measure of risk. For example, if one chooses to measure risk as the downside risk only

$$
\sigma_{n}^{-} \stackrel{\text { def }}{=}\left[\int_{-\infty}^{r} p(x)(r-x)^{n} \mathrm{~d} x\right]^{\frac{1}{n}}
$$


then $\sigma$ is replaced by $\sigma_{n}^{-}$, and the Sharpe ranking function is replaced by the Kappa ranking function [11]

$$
R_{\mathrm{Kappa}-n}(p) \stackrel{\text { def }}{=} \frac{\mu-r}{\sigma_{n}^{-}}
$$

The Kappa ranking function above is the Sortino and Van Der Meer [8] Sortino and Price [9] Sortino and Forsey [10] ranking function when $n=2$ and it is equivalent to the Omega [12] ranking function when $n=1$. A different ranking scheme has been proposed by the Stutzer [14]. For a theoretical analysis of "good" vs "bad" risk measures we refer the reader to the work of Artzner et al. [15].

We show in the last section that these rankings are all equivalent to the Sharpe if returns are Gaussian. If returns are not Gaussian, Sharpe, Sortino, Kappa, and Omega, are not equivalent because they follow from different definitions of risk.

\subsection{CASE \#2: without risk free asset, the wrong way}

In CASE \#2, our investor has to choose a portfolio and invest the entire available funds in it, hence the argument presented at the beginning of the previous section does not apply. The reason behind the use of the Sharpe ranking (or the Sortino ranking) falls apart as pointed out by Sharpe himself [6].

In this subsection we answer two questions:

- Is the use of the Sharpe ranking (or any of the other rankings) however justified?

- If not, what is an appropriate ranking scheme that leads to the correct choice for a rational risk averse investor?

Let's examine first the Sharpe ranking function and assume that portfolio returns are Gaussian distributed. An explicit computation shows that, for every Gaussian portfolio $p$

$$
R_{U_{\text {naive }}}(p) \sim R_{\text {Sharpe }}(p)
$$

where

$$
U_{\text {naive }}(x) \stackrel{\text { def }}{=}\left\{\begin{array}{ll}
-1 & \text { (if } x<0) \\
+1 & (\text { if } x \geq 0)
\end{array}\right\}
$$

and the monotonic mapping function between the two rankings is

$$
h(y)=\operatorname{erf}(y / \sqrt{2})
$$

Therefore, an investor who ranks portfolios using the Sharpe function in CASE \#2 is implicitly adopting the utility function in eq. (10). The problem here is that eq. (10) is not a risk averse utility function. This function says that a positive return $x>0$ (gain) has a utility +1 and a negative return $x<0$ (loss) has a utility -1 . This investor does not think that a $20 \%$ return is better than a $10 \%$ return, or that a 
loss of $100 \%$ is worse than a loss of $1 \%$. The investor that uses the Sharpe ranking function for CASE \#2 is not a rational risk averse investor.

Under the assumption of Gaussian returns Sortino, Omega, Stutzer and Kappa rankings are all equivalent (as proven in the last section), therefore the use of any of these rankings, in the case considered here, is not consistent with being risk averse.

Returns of real portfolios are, generally, non Gaussian distributed but, if a ranking function works for a general distribution, it must work for Gaussian returns too. Since this is not true for Sharpe, Sortino, Omega, Stutzer, nor Kappa, these ranking schemes should not be used in the CASE \#2.

\subsection{CASE \#2: without risk free asset, the right way}

Our investor, a rational risk averse investor, would use the CARA utility function to make choices and would rank portfolios using eq. (2) with $U$ being $U_{C A R A}$. In the last section we prove that $R_{U_{C A R A}}$ is equivalent to $R_{*}$ where

$$
\begin{aligned}
R_{*}(p) & \stackrel{\text { def }}{=}-\log \left(-R_{U_{C A R A}}(p)\right) / m \\
& =\mu-\frac{m \sigma^{2}}{2}+\frac{m^{2} \sigma^{3} S}{6}-\frac{m^{3} \sigma^{4}(K-3)}{720}+O\left(m^{4} \sigma^{5}\right)
\end{aligned}
$$

and

- $\mu$ is the average return of portfolio $p$,

- $\sigma$ is the standard deviation of portfolio $p$,

- $S$ is the skewness,

- $K$ is the kurtosis ( $K-3$ is the reduced kurtosis)

- $m$ is a parameter of the order of 1 , that measures the risk aversion of our investor,

- $O\left(m^{4} \sigma^{5}\right)$ is the order of terms that are ignored.

Notice that a positive skewness is good while a positive reduced kurtosis is bad because it results in fatter tails for fixed $\sigma$.

For Gaussian distributed returns the formula in eq. (12) reduces to

$$
R_{*}(p)=\mu-\frac{m \sigma^{2}}{2}
$$

and it is exact.

Note that the ranking 14 was originally proposed by Levy and Markowitz [16]. Our approximated formula, eq. (13) extends that result in the case of non-Gaussian returns.

We conclude that a rational risk averse investor who has to choose one portfolio among many and has to invest all funds in the selected portfolio, should make its choice based on the ranking function in eq. (12). This is a general result and it does not make any assumption on the distribution of the returns of the portfolios. 


\section{Practical considerations}

Our conclusions have an immediate practical applicability in CASE \#2, i.e. when the investor has to choose one and only one of mutually exclusive portfolios (or investment alternatives) according only to past performance.

Here is an outline of the decision algorithm

- For each portfolio $k$ collect the historical returns $r_{k t}$ (return for portfolio $k$ at time $t$ ) and measure

$$
\begin{aligned}
\mu_{k} & =(1 / N) \sum_{t} r_{k t} \\
\sigma_{k} & =(1 / N) \sum_{t}\left(r_{k t}-\mu_{k}\right)^{2} \\
S_{k} & =(1 / N) \sum_{t}\left(r_{k t}-\mu_{k}\right)^{3} / \sigma_{k}^{3} \\
K_{k} & =(1 / N) \sum_{t}\left(r_{k t}-\mu_{k}\right)^{4} / \sigma_{k}^{4}
\end{aligned}
$$

(where $N$ is the number of available data points)

- For each portfolio compute

$$
\operatorname{Rank}_{k}=\mu_{k}-\frac{m \sigma_{k}^{2}}{2}+\frac{m^{2} \sigma_{k}^{3} S_{k}}{6}-\frac{m^{3} \sigma_{k}^{4}\left(K_{k}-3\right)}{720}
$$

- Sort the portfolios according to $\operatorname{Rank}_{k}$ and select the one with the highest rank.

If $S_{k}=0$ and $K_{k}=0$ our rank is equivalent to the formula proposed by Levy and Markowitz [16].

Notice that the rank depends on whether on the time-scale of our analysis via our choice of returns $r$, which can be daily returns, weekly returns, monthly returns, etc. For a fixed skewness and kurtosis, their relative contribution to the rank increases with the size of the time-scale. In any case, because of the 1/720 factor, the relative contribution of kurtosis is generally very small.

\section{Proofs}

\subsection{Equivalence of ranking schemes for Gaussian distributions}

In this section we prove that if $p(x)$ is a Gaussian distribution with mean $\mu$ and standard deviation $\sigma$, then the Sharpe, Sortino, Omega, Stutzer and Kappa ranking functions are equivalent [5].

Two rankings, $R_{1}$ and $R_{2}$, are equivalent if and only if, for any two portfolios $p_{1}$ and $p_{2}, R_{1}\left(p_{1}\right)<R_{1}\left(p_{2}\right)$ implies $R_{2}\left(p_{1}\right)<R_{2}\left(p_{2}\right)$ and vice versa. This can only occur if there is a monotonic increasing function $h$ such that, for any portfolio $p$, $R_{2}(p)=h\left(R_{1}(p)\right)$. 
Kappa Proof:

$$
R_{\text {Kappa }-n}(p) \stackrel{\text { def }}{=} \frac{\mu-r}{\left[\int_{-\infty}^{r}(r-x)^{n} p(x) \mathrm{d} x\right]^{\frac{1}{n}}}=h\left(R_{\text {Sharpe }}(p)\right)
$$

and

$$
h(y)=\frac{\pi \frac{1}{2 n} 2^{\frac{2-n}{2 n}} e^{\frac{y^{2}}{2 n}} y}{\left[\Gamma\left(\frac{1+n}{2}\right){ }_{1} F_{1}\left(\frac{1+n}{2}, \frac{1}{2}, \frac{y^{2}}{2}\right)-\sqrt{2} \Gamma\left(1+\frac{n}{2}\right) 1 F_{1}\left(1+\frac{n}{2}, \frac{3}{2}, \frac{y^{2}}{2}\right)\right]^{\frac{1}{n}}}
$$

( $\Gamma$ is Euler's Gamma function and ${ }_{1} F_{1}$ is an Hypergeometric function).

Omega Proof:

$$
R_{\text {Omega }}(p) \stackrel{\text { def }}{=} \frac{\int_{r}^{\infty}\left(1-F_{p}(x)\right) \mathrm{d} x}{\int_{-\infty}^{r} F_{p}(x) \mathrm{d} x}=R_{\text {Kappa-1 }}(p)
$$

(here $F_{p}$ is the cumulative distribution function associated to $p$ ).

Sortino Proof:

$$
R_{\text {Sortino }}(p) \stackrel{\text { def }}{=} \frac{\mu-r}{\left[\int_{-\infty}^{r}(r-x)^{2} p(x) \mathrm{d} x\right]^{\frac{1}{2}}}=R_{\mathrm{Kappa}-2}(p)
$$

Stutzer Proof:

$$
R_{\text {Stutzer }}(p) \stackrel{\text { def }}{=} \lim _{T \rightarrow \infty} \frac{-\log F_{p}(r T)}{T}=h\left(R_{\text {Sharpe }}(p)\right)
$$

$R_{\text {Stutzer is }}$ well defined only for portfolios with positive Sharpe ratio, and

$$
h(y)=y^{2} / 2 \text { (for } y>0 \text { only) }
$$

\subsection{Equivalence of rankings skemes in presence of skewness and kurtosis}

In this section we prove that $R_{U_{C A R A}}$ is equivalent to $R_{*}$.

Consider a portfolio characterized by a distribution of returns $p$. Let $\mu, \sigma, S, K$ be the average, standard deviation, skewness and kurtosis of $p$.

$$
R_{U_{C A R A}}(p) \stackrel{\text { def }}{=} \int_{-\infty}^{\infty}-e^{-m x} p(x) \mathrm{d} x=\int_{-\infty}^{\infty}-e^{-m x} \tilde{p}((x-\mu) / \sigma) \mathrm{d} x
$$

where $\tilde{p}(y)=p(\sigma y+\mu) \sigma$. With the change of variable $y=(x-\mu) / \sigma$ and a Taylor series expansion in $y$ of the exponential we obtain

$$
\begin{aligned}
R_{U_{C A R A}}\left(p_{i}\right) & =-e^{-m \mu} \sum_{i=0} \frac{(-m \sigma)^{i}}{i !} \int_{-\infty}^{\infty} y^{i} \tilde{p}(y) \mathrm{d} y \\
& =-e^{-m \mu} e^{\log \left(1+\frac{m^{2} \sigma^{2}}{2}-\frac{m^{3} \sigma^{3} S}{3 !}+\frac{m^{4} \sigma^{4}(K-3)}{6 !}+O\left(m^{4} \sigma^{5}\right)\right)} \\
& =h\left(R_{*}(p)\right)
\end{aligned}
$$


where $h(y)=-e^{-m y}$ is monotonic increasing function in $y$, and

$$
\begin{aligned}
R_{*}(p) & \stackrel{\text { def }}{=}-\log \left(-R_{U_{C A R A}}(p)\right) / m \\
& =\mu-\frac{m \sigma^{2}}{2}+\frac{m^{2} \sigma^{3} S}{6}-\frac{m^{3} \sigma^{4}(K-3)}{720}+O\left(m^{4} \sigma^{5}\right)
\end{aligned}
$$

Hence the two ranking functions $R_{*}(p)$ and $R_{U_{C A R A}}(p)$ are equivalent. This is a general result and no assumption about the distribution $p$ has been made.

In the special case of $p$ Gaussian, we are able to perform the integration analytically without need for a Taylor expansion and we find that the following exact relation holds [16],

$$
R_{*}(p)=\mu-\frac{m \sigma^{2}}{2}
$$

\section{Conclusions}

In this paper we discuss the issue of portfolio selection for a rational risk averse investor. We consider two cases: the investor is free to distribute funds between the selected portfolio and a risk free asset; and the case when the investor has to invest all funds in the selected portfolio.

In the first case we find that Sharpe, Omega, Sortino, and Kappa provide valid ranking schemes, although they follow from different definitions of risk, $\sigma, \sigma_{1}^{-}$, $\sigma_{2}^{-}$and $\sigma_{n}^{-}$respectively. We also find that, in the Gaussian case, Sharpe, Omega, Sortino, Kappa, and Stutzer rankings are all equivalent. In the non-Gaussian case these rankings are not equivalent $[15,1]$.

In the second case we find that, contrary to what is sometimes claimed, the use of any of the above ranking schemes does not correspond to being a rational risk averse investor. In fact, we prove that a rational risk averse investor (compatible with the choice of the CARA utility function) would choose a portfolio according to the ranking function in eq. (1), which takes into account the skewness of the portfolio, $S$, and its kurtosis, $K$. Our formula, eq. (1), extends a result originally due to Levy and Markowitz [16] in the case of non-Gaussian returns.

\section{References}

[1] S. Ortobelli, S. T. Rachev, S. Stoyanov, F. Fabozzi, and A. Biglova, "The Proper Use of Risk Measures in Portfolio Theory", International Journal of Theoretical and Applied Finance, Dec 2005.

[2] P. A. Horvath and R. C. Scott, "On the direction of preference for moments of higher order than variance", Journal of Finance 35, 1980, pp. 915-919.

[3] Neumann, John von and Morgenstern, Oskar, Theory of Games and Economic Behavior. Princeton, NJ. Princeton University Press. 1944 sec.ed. 1947.

[4] Nash Jr., John F. The Bargaining Problem. Econometrica 18:155 1950. 
[5] M. Di Pierro and J. Mosevich, "On Ranking Schemes and Portfolio Selection", published in "Hedge Funds and Investment Management", I. Nelken Ed., Butterworth-Heinemann publisher.

[6] W. F. Sharpe, "Capital asset prices: A theory of market equilibrium under conditions of risk", Journal of Finance, 1964, 19(3), pp. 425-442.

[7] H. M. Markowitz, "Portfolio Selection", Journal of Finance 1952, 7 (1), pp. 77-91.

[8] F. A. Sortino and R. Van Der Meer, "Downside Risk", Journal of Portfolio Management, 1991, v17(4), 27-32.

[9] F. A. Sortino and L. N. Price, "Performance Measurement In A Downside Risk Framework", Journal of Investing, 1994, v3(3), 59-64.

[10] F. A. Sortino and H. J. Forsey, "On The Use And Misuse Of Downside Risk", Journal of Portfolio Management, 1996, v22(2,Winter), 35-42.

[11] P. D. Kaplan and J. A. Knowles, "Kappa: A Generalized Downside RiskAdjusted Performance Measure", Journal of Performance Measurement, Vol. 8, \#3 - Spring 2004.

[12] H. Kazemi, T. Schneeweis and R. Gupta, "Omega as Performance Measure" CISDM 2003 Proceedings.

[13] F. A. Sortino, "From Alpha to Omega", in Managing Downside Risk in Financial Markets, F. A. Sortino and S. E. Satchell, eds., Reed Educational and Professional Publishing Ltd., 2001.

[14] M. Stutzer, “A Portfolio Performance Index", Financial Analysts Journal, Vol. 56, May-June 2000.

[15] P. Artzner, F. Delbaen, J. M. Heath, and D. Eber, "Coherent measures of Risk", Mathematical Finance 9, 2000, pp. 203-228.

[16] H. Levy and H. Markowitz, "Approximating expected utility by a function of mean and variance”, American Economic Review 69, 1979, pp. 308-317. 